ARTICLE

DOI: $10.1038 / s 41467-018-06904-9$

\title{
syn-Selective alkylarylation of terminal alkynes via the combination of photoredox and nickel catalysis
}

Lei Guo ${ }^{1}$, Fan Song ${ }^{1}$, Shengqing Zhu', Huan $\mathrm{Li}^{1} \&$ Lingling Chu ${ }^{1}{ }^{1}$

Substituted alkenes are pivotal structural motifs found in pharmaceuticals and agrochemicals. Although numerous methods have been developed to construct substituted alkenes, a generally efficient, mild, catalytic platform for the conversion of alkynes to this highly functionalized scaffold via successive $\mathrm{C}-\mathrm{C}$ bond forming steps remains in high demand. Here we describe an intermolecular, regio- and syn-stereoselective alkylarylation of terminal alkynes with tertiary alkyl oxalates via photoredox-Ni dual catalysis. This catalytic protocol, synergistically combining Ir/Ni-catalyzed alkyne difunctionalization with photoinduced alkene isomerization, affords trisubstituted alkenes with excellent efficiency and syn-stereoselectivity. The mild conditions tolerate many functional groups, allowing for a broad scope with respect to terminal alkynes, aryl bromides, and alkyl oxalates.

\footnotetext{
${ }^{1}$ State Key Laboratory for Modification of Chemical Fibers and Polymer Materials, College of Chemistry, Chemical Engineering and Biotechnology, Center for Advanced Low-Dimension Materials, Donghua University, Shanghai 201620, China. Correspondence and requests for materials should be addressed to L.C. (email: lingling.chu1@dhu.edu.cn)
} 
S ubstituted alkenes are pivotal structural motifs found in pharmaceuticals, agrochemicals, and biologically active natural products ${ }^{1-3}$, as well as versatile synthetic building blocks in organic synthesis ${ }^{4,5}$. Consequently, the development of general protocols for the regio- and stereoselective synthesis of alkenes, particularly employing readily available and abundant precursors, is a long-standing goal of chemical synthesis 6,7 . Transition metal-catalyzed direct functionalization of alkynes is a powerful method to access substituted alkenes with stereoselectivity control ${ }^{8-21}$. Generally, two distinct strategies have been utilized: one is a catalytic generation of syn-substituted alkenyl metal species, via migratory insertion controlled by the stereoelectronic effect, followed by protonation or coupling to deliver trisubstituted alkenes with $s y n$-stereoselectivity ${ }^{8-28}$; the other is transition metal-catalyzed radical addition/coupling reaction of alkynes, typically favoring anti-addition which is dominated by steric factors of vinyl radicals in the coupling step (Fig. 1a) ${ }^{29-37}$. Although numerous methods have been developed toward addressing the challenge of regio- and stereo-selectively forming trisubstituted alkenes, a generally efficient, mild, catalytic platform for the conversion of alkynes to this highly functionalized scaffold via successive $\mathrm{C}-\mathrm{C}$ bond forming steps would be highly demanding and represent a valuable advance in synthetic methodology.

Over the last decade, visible light photocatalysis have emerged as a powerful platform in organic synthesis by activating organic molecules through either single-electron transfer or energy transfer $^{38-43}$. Particularly, the ability of the photoredox catalyst to modulate the oxidation state of organometallic species has enabled the efficient construction of challenging C-C bonds ${ }^{44-49}$. Recently, this solar-energy-driven catalytic technology has been utilized to facilitate contra-thermodynamic $E \rightarrow Z$ isomerization of olefins through an energy-transfer manifold, enabling the facile synthesis of $Z$-olefins ${ }^{50-56}$. We recently questioned whether metallaphotoredox catalysis could serve as an alternative platform to access trisubstituted alkenes with high control over stereoselectivity ${ }^{57-59}$. Specifically, a catalytic protocol including three sequential events, (i) alkyl radical addition to the $\mathrm{C} \equiv \mathrm{C}$ bond ${ }^{29}$, (ii) cross-coupling of the resulting alkenyl radical with nickel complex, and (iii) photochemical $E \rightarrow Z$ isomerization of olefins, would deliver the stereodefined trisubstituted alkenes. We envisioned that the unique metallaphotoredox manifold would enable the generation of alkyl radicals from readily available feedstocks ${ }^{60}$ such as alcohols ${ }^{61}$, and more importantly could be leveraged to enrich the stereoselectivity of alkyne addition reactions. Herein, we demonstrate the first example of alkylarylation of terminal alkynes employing simple tertiary alcohol derivatives and aryl halides through the synergistic merger of photoredox and nickel catalysis ${ }^{62,63}$, furnishing a wide array of trisubstituted alkenes with syn-stereoselectivity under mild conditions (Fig. 1b). This photoredox protocol provides complementary reactivity and stereoselectivity to a previous nickel system with alkyl halides as radical precursors, which affords the trisubstituted alkenes with anti-stereoselectivity at elevated temperature $\left(80-120^{\circ} \mathrm{C}\right)^{34}$.

\section{Results}

Design plan. Inspired by Overman and MacMillan's oxalate halfester chemistry ${ }^{64}$, we chose tertiary alkyl oxalates as the alkyl precursors for our proposed metallaphotoredox alkyne chemistry. Tertiary oxalate salts are bench stable, and can be easily prepared from corresponding, abundant tertiary alcohols. As depicted in Fig. 2, we envisioned that a single-electron oxidation of tertiary alkyl oxalate $3\left(E_{1 / 2}=+1.28 \mathrm{~V}\right.$ vs SCE in $\mathrm{CH}_{3} \mathrm{CN}$ for tertBuOCOCO $\left.{ }_{2} \mathrm{Cs}\right)^{64}$ by photoexcited $\operatorname{Ir}\left[\mathrm{dF}\left(\mathrm{CF}_{3}\right) \text { ppy }\right]_{2}(\mathrm{dtbbpy})\left(\mathrm{PF}_{6}\right)$ $1\left(E_{1 / 2}\left[\mathrm{Ir}^{*} \mathrm{III} / \mathrm{Ir}^{\mathrm{II}}=+1.21 \mathrm{~V} \text { vs SCE in } \mathrm{CH}_{3} \mathrm{CN}\right]^{65}\right.$ should generate alkyl radical 4 upon loss of two molecules of $\mathrm{CO}_{2}$ as well as reduced $\operatorname{Ir}(\mathrm{II})$ species $\mathbf{5}$. Alkyl radical $\mathbf{4}$ is expected to undergo regioselective addition to terminal alkyne $\mathbf{6}$ to produce linearized alkenyl radical 7 due to resonance stabilization effect ${ }^{29}$. An antiaddition of the high-energy alkenyl radial 7 and $\mathrm{Ni}(0) \mathbf{8}$ is expected to deliver the $(E)$-alkenyl-Ni(I) species $\mathbf{9}^{66}$. Subsequent

a Previous work: transition metal-catalyzed functionlization of alkynes

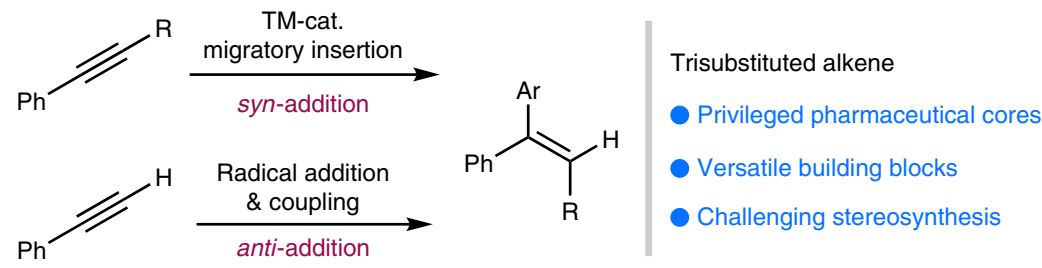

b This work: syn-alkylarylation of alkynes via metallaphotoredox catalysis
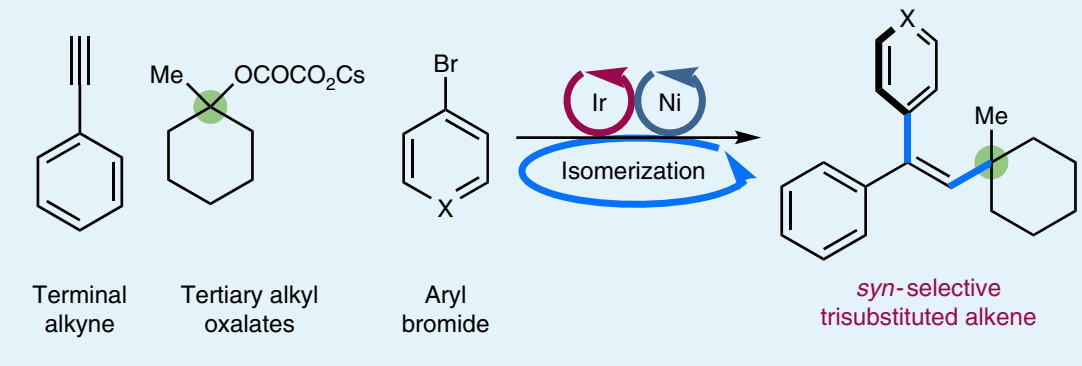

Readily available materials

Chemo \& regio \& stereoselective

Consecutive construction of two $\mathrm{C}-\mathrm{C}$ bonds \& alkene isomerization

Fig. 1 syn-Alkylarylation of terminal alkynes via the combination of photoredox and nickel catalysis. a Alkyne functionalizations via transition metal catalysis. $\mathbf{b}$ syn-Alkylarylation of alkynes via metallaphotoredox catalysis 


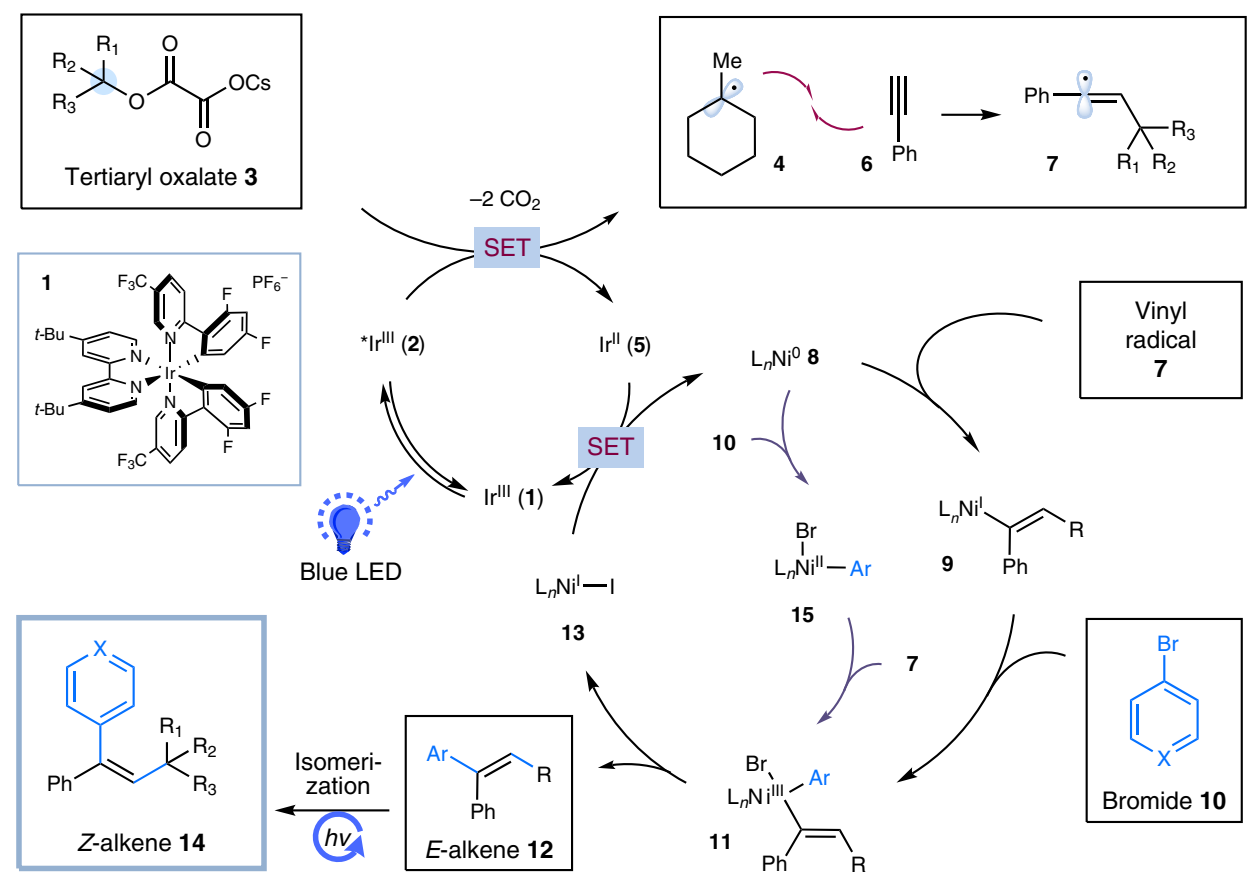

Fig. 2 Proposed mechanism. Two possible reaction pathways proposed on the basis of previous literature

oxidative addition of (E)-alkenyl-Ni(I) 9 with aryl bromide $\mathbf{1 0}$ would afford (E)-alkenyl-Ni(III) complex $\mathbf{1 1}^{34,67}$, which undergoes a facile reductive elimination to produce substituted alkene 12 with concomitant generation of $\mathrm{Ni}(\mathrm{I})$ complex 13. Singleelectron transfer between $\operatorname{Ir}(\mathrm{II}) \mathbf{5}\left\{E_{1 / 2}\left[\mathrm{Ir}^{\mathrm{III}} / \mathrm{Ir}^{\mathrm{II}}=-1.37 \mathrm{~V}\right.\right.$ vs SCE in $\left.\left.\mathrm{CH}_{3} \mathrm{CN}\right]\right\}^{65}$ and $\mathrm{Ni}(\mathrm{I}) \mathbf{1 3}\left(E_{1 / 2}\left[\mathrm{Ni}^{\mathrm{II}} / \mathrm{Ni}^{0}=-1.2 \mathrm{~V}\right.\right.$ vs $\mathrm{SCE}$ in $D_{M M F}{ }^{68}$ would regenerate ground-state $\operatorname{Ir}(\mathrm{III}) \mathbf{1}$ and $\mathrm{Ni}(0)$ to close the two catalytic cycles. Given the polarity of $\mathbf{1 2}$, at this juncture, we hypothesized that a $E \rightarrow Z$ isomerization of $\mathbf{1 2}$ would be possible through a photoinduced energy transfer manifold ${ }^{50-56}$, delivering the desired alkene 14 with syn-stereoselectivity. Alternatively, another catalytic pathway involving oxidative addition of $\mathrm{Ni}(0)$ with aryl bromide $10^{62,63,69}$, followed by trapping of the nucleophilic vinyl radical 7 by aryl-Ni(II) 15 to furnish the key $\mathrm{Ni}(\mathrm{III})$ intermediate 11, is also plausible.

Optimization study. We examined the feasibility of this proposed metallaphotoredox alkyne protocol by employing 4-tertbutylphenylacetylene 16 and 4-bromobenzaldehyde 17 as standard substrates. As shown in Table 1, upon irradiation by a $90 \mathrm{~W}$ blue LED of a solution of alkyne 16, bromide 17, and cesium oxalate 18 derived from 1-methyl-1-cyclohexanol in the presence of catalytic amounts of $\operatorname{Ir}\left[\mathrm{dF}\left(\mathrm{CF}_{3}\right) \text { ppy }\right]_{2}(\mathrm{dtbbpy}) \mathrm{PF}_{6} \mathbf{1}, \mathrm{NiCl}_{2} \bullet-$ glyme, 4,4'-di-tert-butyl-2,2'-dipyridyl (dtbbpy) in DMSO, $81 \%$ yield of the desired trisubstituted alkene product 19 was obtained with excellent chemo-, regio-, and syn-selectivity $(Z / E=96: 4)$ (entry 1). The structurally similar photocatalyst $\operatorname{Ir}\left[\mathrm{dF}\left(\mathrm{CF}_{3}\right)\right.$ ppy $]_{2}$ (phen) $\mathrm{PF}_{6}$ also promoted this transformation with moderate efficiency and excellent syn-selectivity (entry 2). Switching to other commonly employed photocatalysts, such as $\mathrm{Ru}$ (bpy $)_{3}\left(\mathrm{PF}_{6}\right)_{2}$ and 4CzlPN (2,4,5,6-tetra(9H-carbazol-9-yl)isophthalonitrile), resulted in a dramatic decrease in efficiency (entries 3-4). The reaction proceeded with moderate to good efficiency in the presence of $\mathrm{NiCl}_{2}\left(\mathrm{PPh}_{3}\right)_{2}$ or precatalyst $\mathrm{NiCl}_{2}(\mathrm{Py})_{4}$ (entries 5-6). The choice of solvent demonstrated a

\section{Table 1 Optimization of reaction conditions. ${ }^{a}$}

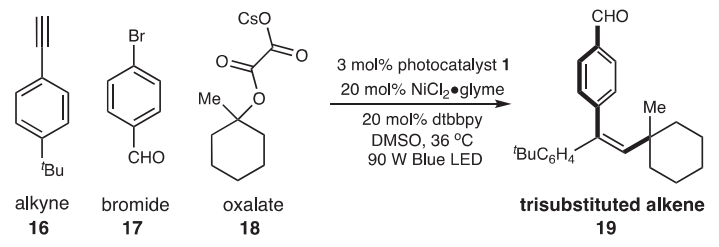

$\begin{array}{llll}\text { Entry } & \text { Variations from standard conditions } & \text { Yield } & \mathrm{Z} / \mathrm{E} \\ 1 & \text { None } & 81 \% & 96: 4 \\ 2 & \mathrm{Ir}\left[\mathrm{dF}\left(\mathrm{CF}_{3}\right) \mathrm{ppy}\right](\mathrm{dtbbpy})\left(\mathrm{PF}_{6}\right) & 56 \% & 97: 3 \\ 3 & \mathrm{Ru}(\mathrm{bpy})_{3}\left(\mathrm{PF}_{6}\right)_{2} & 10 \% & - \\ 4 & 4 \mathrm{CzlPN} & 8 \% & - \\ 5 & \mathrm{NiCl}_{2}\left(\mathrm{PPh}_{3}\right)_{2} & 61 \% & 96: 4 \\ 6 & \mathrm{NiCl}_{2}\left(\mathrm{Py}_{4}\right. & 80 \% & 97: 3 \\ 7 & \mathrm{DMA}_{3} & 54 \% & 96: 4 \\ 8 & \mathrm{DMF} & 74 \% & 96: 4 \\ 9 & \mathrm{CH} \mathrm{H}_{3} \mathrm{CN} & 45 \% & 95: 5 \\ 10 & \text { DME } & 40 \% & 96: 4 \\ 11 & \text { No photocatalyst } & 0 \% & - \\ 12 & \text { No nickel catalyst } & 0 \% & - \\ 13 & \text { No ligand } & 0 \% & - \\ 14 & \text { No light } & 0 \% & -\end{array}$

aReaction conditions: photocatalyst ( $3 \mathrm{~mol} \%$ ), $\mathrm{NiCl}_{2} \cdot \mathrm{glyme}(20 \mathrm{~mol} \%$ ), dtbbpy $(20 \mathrm{~mol} \%$ ), alkyne $(0.1 \mathrm{mmol})$, oxalate $(1.5$ equiv.), aryl bromide (2.0 equiv.), DMSO [0.05 M], $90 \mathrm{~W}$ blue LED, $36^{\circ} \mathrm{C}, 18 \mathrm{~h}$. Yields determined by ${ }^{1} \mathrm{H}$ NMR with an internal standard, and the ratio of the two isomers was determined by ${ }^{1} \mathrm{H}$ NMR analysis of the crude reaction mixture. dtbbpy $=4,4^{\prime}$ di-tert-butyl-2,2'-dipyridyl; 4 CzIPN = 2,4,5,6-tetra( $9 H$-carbazol-9-yl)isophthalonitrile 


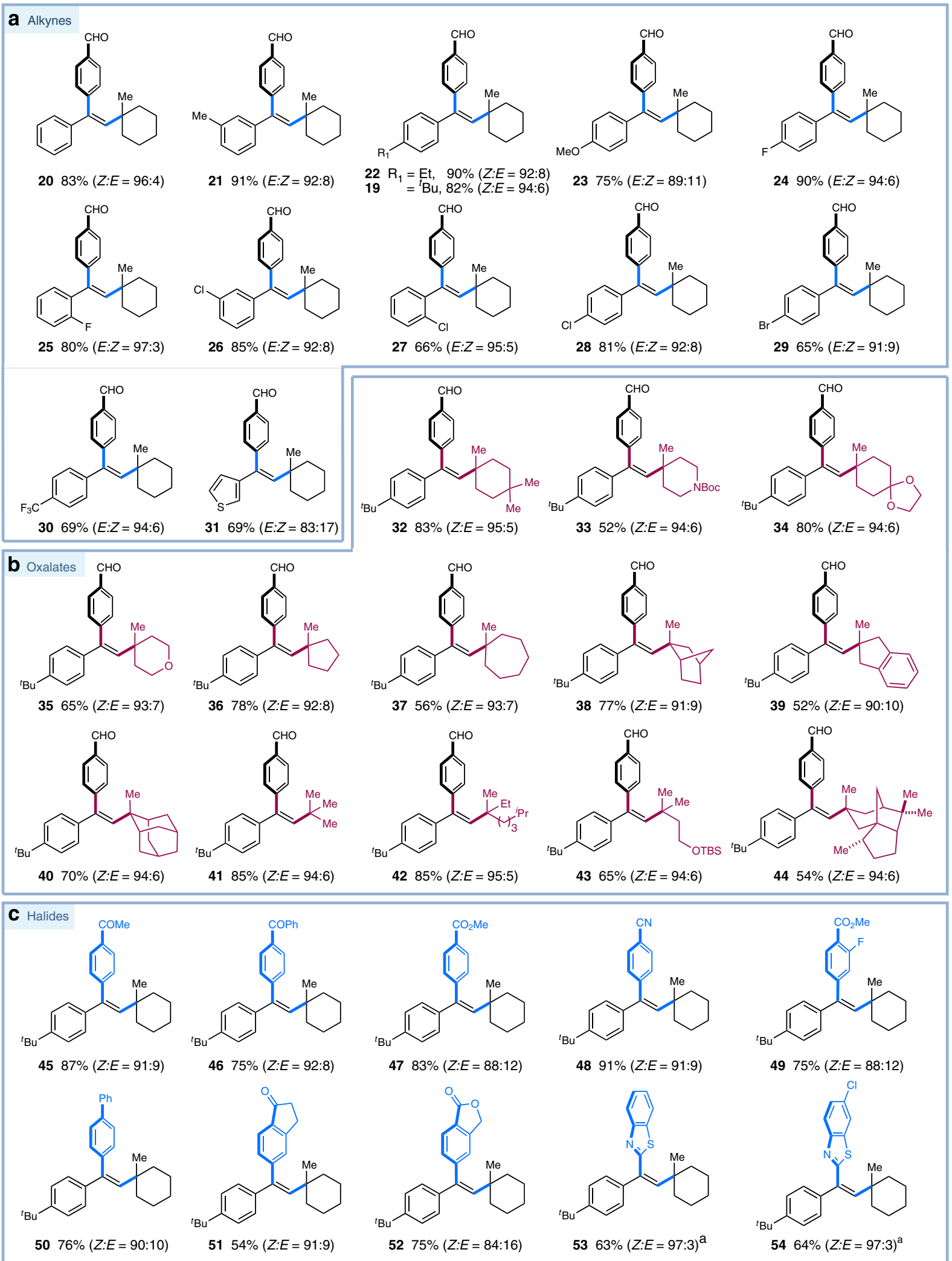

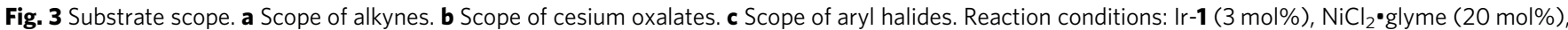
dtbbpy (20 mol\%), alkyne $(0.1 \mathrm{mmol})$, oxalate (1.5 equiv.), bromide (2.0 equiv.), DMSO [0.05 M], $90 \mathrm{~W}$ blue LED, $36^{\circ} \mathrm{C}, 18 \mathrm{~h}$. All cited yields are isolated

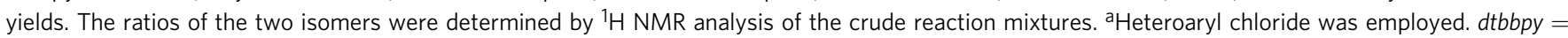
4,4'-di-tert-butyl-2,2'-dipyridyl 
dramatic effect on the reaction efficiency, with DMSO proving to be optimal (entries 7-10). Interestingly, stereoselectivity of the alkene product appeared to be solvent independent (entries 7-10). Finally, control experiments demonstrated that light, photocatalyst, nickel catalyst, and ligand are all essential for the desired transformation to proceed (entries 11-14) (for additional control experiments, see Supplementary Tables 1,2).

Substrate scope. With optimal conditions in hand, we explored the generality of this metallaphotoredox protocol with respect to the alkyne fragment. As depicted in Fig. 3a, terminal arylalkynes bearing electron-neutral, -donating, and -withdrawing substituents proceeded smoothly under the optimal conditions, furnishing the corresponding trisubstituted alkenes with high to excellent yields and stereoselectivity (products 19-30, 65-90\% yields, $>95: 5$ syn-selectivity). Notably, halides ( $\mathrm{F}, \mathrm{Cl}, \mathrm{Br}$ ) on the aryl ring of alkynes remained untouched, offering valuable handles for further manipulations (product 24-29, 65-90\% yields, $>91: 9$ syn-selectivity). Moreover, ortho substituents on the aryl ring had little effect to the reaction efficiency and selectivity (products 25 and $27,80 \%$ and $66 \%$ yield, $>95: 5$ synselectivity, respectively). A slight erosion of yield and selectivity was observed when 3-ethynylthiophene was subjected to this multicomponent system (products 31, 69\% yield, 83:17 synselectivity). However, internal alkynes are not compatible in this reaction protocol, a result we attribute to increased steric hindrance.

Next, we evaluated the scope of cesium tertiary alkyl oxalates in this protocol. As shown in Fig. 3b, a variety of tertiary cesium oxalates, readily prepared from the corresponding tertiary alcohols, can be successfully employed with high levels of efficiency and stereoselectivity. Cyclic oxalates, derived from cyclohexanols, cyclopentanols, and cycloheptanols, underwent the desired addition/coupling smoothly, yielding the $(Z)$-selective trisubstituted alkenes with high efficiency (products $\mathbf{3 2}-\mathbf{4 0}$ and 44, $52-83 \%$ yields, $Z / E$ up to $95: 5$ ). Heterocycles, in the form of tetrahydropyran and piperidine, were also viable substrates (products 33 and 35, 52\% and 65\% yields, $Z / E>93: 7$ ). A number of polycyclic oxalates could be effectively employed without loss in yield and selectivity (products $\mathbf{3 4}, \mathbf{3 8}-\mathbf{4 0}$, and $\mathbf{4 4}, 49-84 \%$ yields, $Z / E$ up to 94:6). Moreover, this photoredox protocol could further be applied to acyclic tertiary oxalates, furnishing the desired alkenes in good yields and excellent stereoselectivity favoring syn-addition (products 41-43, 65-85\% yields, $Z / E>$ 94:6). Notably, natural-product-derived substrates, such as cedrol oxalate, proved successful, indicating the potential utility of this mild protocol with complex molecules (products $44,54 \%$ yield, $Z / E=94: 6)$.

Finally, we turned our attention to the scope of aryl bromides that can participate in this catalytic protocol. As revealed in Fig. 3c, a variety of electron-deficient aryl bromides can be readily employed with high efficiency and moderate-to-high stereose-

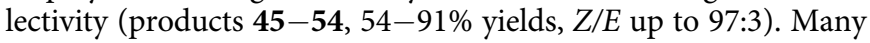
valuable functional groups, including ketones, esters, nitrile, and lactones were found to be well tolerated under the mild conditions (products $\mathbf{4 5 - 5 2}, 54-91 \%$ yields, $Z / E$ up to $92: 8$ ). Gratifyingly, benzothiazole-derived heteroaromatic chlorides could be efficiently employed in this synergistic protocol without any loss in stereoselectivity, albeit with a slight decrease in yields (products $\mathbf{5 3}$ and $\mathbf{5 4}, 63 \%$ and $64 \%$ yields, $Z / E=97: 3$, respectively). At this stage in our studies on this metallaphotoredox protocol, the scope of aryl halides is currently limited to electron-poor and electron-neutral system, in which a conjugated substituent at the para position is crucial to achieve excellent synstereoselectivity control (see Supplementary Figs. 13, 14).

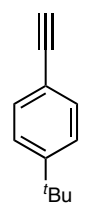

$0.63 g$

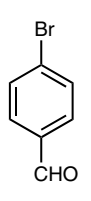

$0.74 \mathrm{~g}$

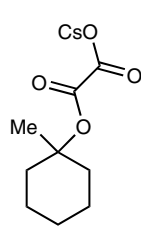

$1.27 \mathrm{~g}$

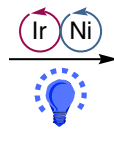

${ }^{t} \mathrm{Bu}$

$19,65 \%$ yield $(Z: E>98: 2)$
Fig. 4 Reaction on large scale. Reaction condition: Ir-1 ( 3 mol\%), $\mathrm{NiCl}_{2} \bullet$ glyme (20 mol\%), dtbbpy (20 mol\%), alkyne ( $4 \mathrm{mmol})$, oxalate (1.5 equiv.), bromide (2.0 equiv.), DMSO [0.05 M], $90 \mathrm{~W}$ blue LED, $36^{\circ} \mathrm{C}, 18 \mathrm{~h}$. Isolated yield. The ratio of the two isomers were determined by ${ }^{1} \mathrm{H}$ NMR analysis of the crude reaction mixture. dtbbpy $=4,4^{\prime}$-di-tert-butyl-2,2'-dipyridyl

To highlight the synthetic utility of this metallaphotoredox difunctionalization manifold, a gram-scale reaction of alkyne 16 was performed. The reaction proceeded smoothly, affording the desired alkylarylation product 19 in $65 \%$ yield with excellent stereoselectivity (Z/E $>98: 2)$ (Fig. 4).

Mechanistic studies. To probe the reaction pathway of this metallaphotoredox three-component coupling protocol, we conducted a series of preliminary mechanistic experiments. Time course studies showed that yields steadily increased overtime, while high stereoselectivity was observed at the early stage, suggesting that stereoselectivity of this transformation might be catalyst-independent (Fig. 5a). To probe the stereo-enrichment process, $(E)$-alkene $\mathbf{4 1}$ was prepared and subjected to the photoinduced system (Fig. $5 c$ ). In the presence or absence of photocatalyst 1, $(E)-\mathbf{4 1}$ underwent the isomerization to yield $(Z)-\mathbf{4 1}$ with excellent efficiency, indicating that photocatalyst does not actually engage in the isomerization event (Fig. 5c). As expected, no isomerization of $\mathbf{4 1}$ was observed in the absence of visible light (Fig. 5c). On the basis of these results, we expected that trisubstituted alkene itself might act as a photosensitizer for this photochemical isomerization. This hypothesis was further confirmed by UV-Vis absorption spectrometry of $(E)$-41, which exhibited considerable overlap with the blue LED spectrum (Fig. 5b). Particularly, the $(E)$-isomer showed strong bathochromic shift relative to the (Z)-isomer (Fig. 5b). Predictably, deconjugation of the $\pi$-system in the $(Z)$-isomer product secures high levels of stereocontrol in this contra-thermodynamic, photoinduced $E \rightarrow$ $Z$ isomerization (see Supplementary Fig. 3 for density functional theory (DFT)-based conformational analysis). Furthermore, reaction of oxalate 18 with alkyne 16 in the absence of aryl bromide afforded the alkene product $\mathbf{5 5}$ in 23\% yield (Fig. 5d), providing supporting for the addition of alkyl radical to alkyne step shown in Fig. 2. Nevertheless, only a trace amount of tertalkyl-aryl coupling product was observed in the reaction of oxalate 18 with aryl bromide 17 (see Supplementary Fig. 10). These results suggest that, under these metallaphotoredox conditions, tertiary alkyl radical would be more prone to undergo radical addition to alkyne, as opposed to capture by nickel species, probably due to steric hindrance ${ }^{70}$. Finally, we have prepared Ni (II) oxidative addition complex ${ }^{69,71} \mathbf{5 6}$ to evaluate an alternative pathway involving radical capture by aryl-Ni(II) species $\mathbf{1 5}$ (Fig. 2). Irradiation with a $90 \mathrm{~W}$ blue LED of a solution of isolated aryl- $\mathrm{NiI}^{\mathrm{II}}-\mathrm{Br} 56$, alkyne 16, and oxalate 18 in the presence of stoichiometric amount of photocatalyst $\mathbf{1}$ led to no formation of the desired trisubstituted alkene product (Fig. 5e). The major byproduct was biaryl, which could be formed via homo-coupling from disproportionation of $\mathrm{Ni}(\mathrm{II})$ complex 56 (see Supplementary Fig. 12 and Supplementary Table 4). Therefore, we expected that a catalytic $\mathrm{Ni}^{\mathrm{i} / / / / I I I}$ pathway, proceeding via capture of alkenyl 


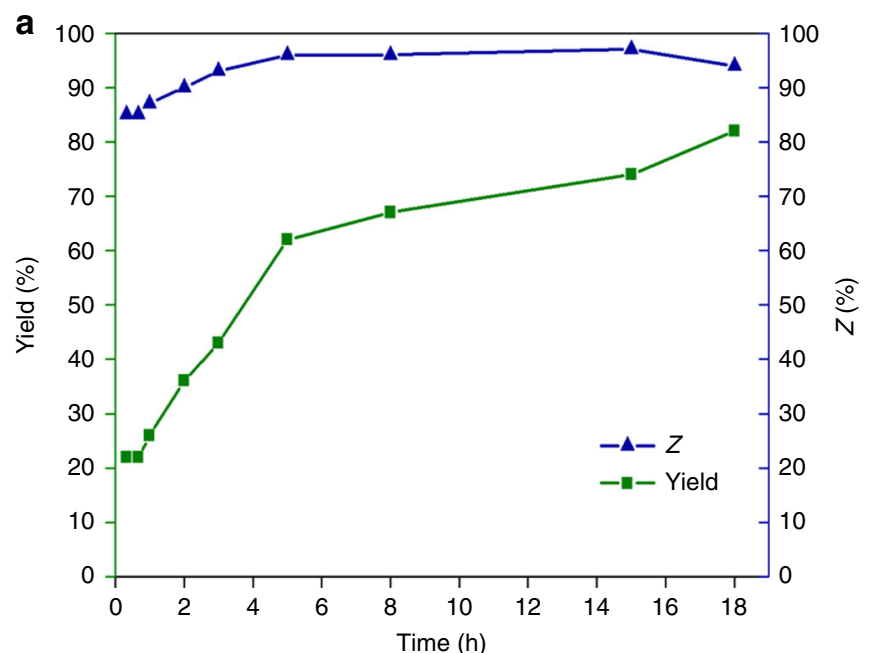

C

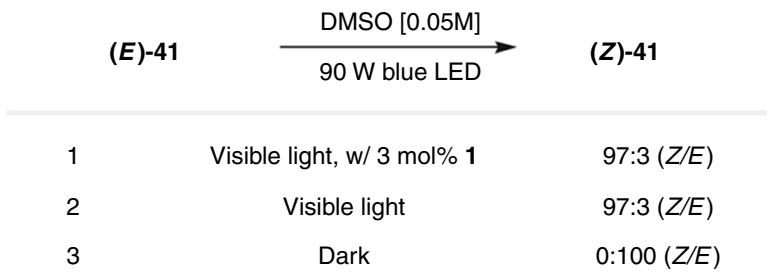

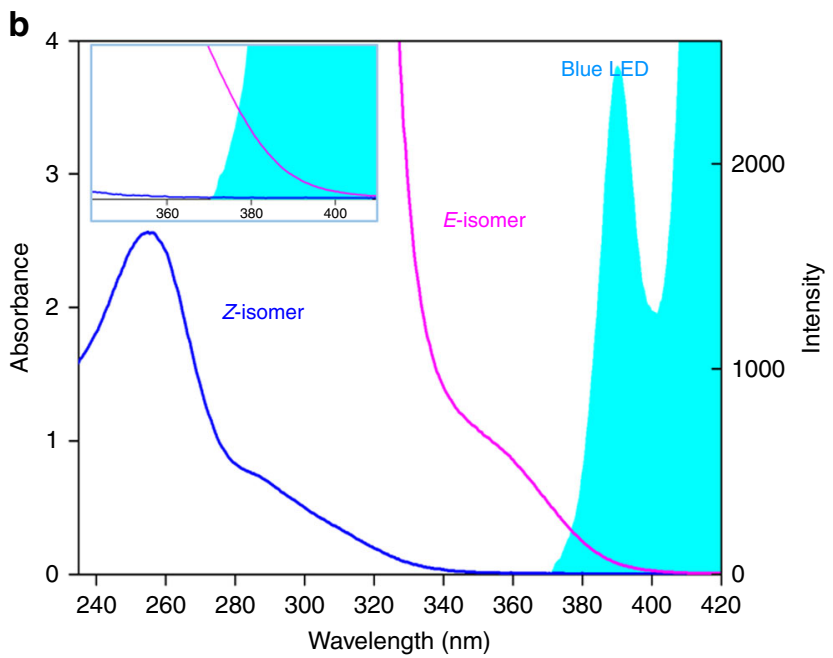

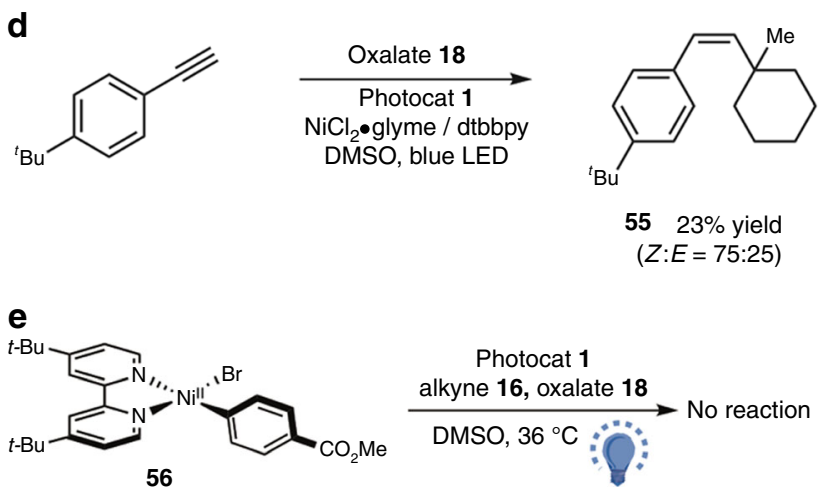

Fig. 5 Mechanistic studies. a Time course studies; b UV-Vis absorption spectrometry; c Isomerization experiments; d Reaction of alkyne with oxalate; e Stoichiometric reaction of isolated $\mathrm{Ni}(\mathrm{II})$ complex. $d$ tbbpy $=4,4^{\prime}$-di-tert-butyl-2,2'-dipyridyl

radical by $\mathrm{Ni}(0)$, followed by oxidative addition and then reductive elimination, could be operative in this metallaphotoredox manifold (Fig. 2).

\section{Discussion}

In conclusion, we have developed a generic protocol for the intermolecular, regioselective, syn-alkylarylation of terminal alkynes with tertiary alkyl oxalates through a synergistic merger of photoredox and nickel catalysis. A one-pot, three-step sequence, involving radical addition, transition-metal-based coupling, and alkene isomerization, proceeds with high efficiency under the light-induced mild conditions. This manifold forges two vicinal C-C bonds, yielding a variety of trisubstituted alkenes with excellent regioselectivity and syn-stereoselectivity. We expect that the operational simplicity and generality of this methodology and readily availability of the starting materials will allow it to enjoy extensive application in the area of organic chemistry.

\section{Methods}

General procedure for the syn-selective alkylarylataion reaction. To a flame dried $8 \mathrm{~mL}$ reaction vial was charged with $\left.\operatorname{Ir}\left[\mathrm{dF}_{(\mathrm{CF}}\right) \mathrm{ppy}\right]_{2}(\mathrm{dtbbpy})\left(\mathrm{PF}_{6}\right)$ (0.003 mmol, $3 \mathrm{~mol} \%), \mathrm{NiCl}_{2} \bullet \mathrm{DME}$ (0.02 mmol, $\left.20 \mathrm{~mol} \%\right), 4,4^{\prime}$-di-tert-butyl-2,2'dipyridyl $(0.02 \mathrm{mmol}, 20 \mathrm{~mol} \%)$, aryl bromide $(0.2 \mathrm{mmol}, 2.0$ equiv.), and cesium alkyl oxalate $(0.15 \mathrm{mmol}, 1.5$ equiv. $)$. The vial was capped. After evacuated and backfilled nitrogen three times, DMSO $[0.05 \mathrm{M}]$ was added via a syringe, followed by the addition of terminal alkyne ( $0.1 \mathrm{mmol}, 1.0$ equiv.). The reaction mixture was irritated with a $90 \mathrm{~W}$ blue LED, with cooling from a fan $\left(36^{\circ} \mathrm{C}\right)$. After $18 \mathrm{~h}$, the reaction was quenched with $\mathrm{H}_{2} \mathrm{O}$, extracted with ethyl acetate. The combined organic layers were dried with $\mathrm{MgSO}_{4}$, filtered, and concentrated in vacuo. The crude material was purified by flash chromatography to afford the products. See Supplementary Methods for further experimental details.

\section{Data availability}

The authors declare that all the data supporting the findings of this work are available within the article and its Supplementary Information files, or from the corresponding author upon request.

Received: 17 July 2018 Accepted: 25 September 2018

Published online: 31 October 2018

\section{References}

1. Stewart, J., Charest, M.-P. \& Herr, F. A pharmacological investigation of potential antidepressants of the amitriptyline-type. J. Med. Chem. 6, 338-339 (1963).

2. Jordan, V. C. Antiestrogens and selective estrogen receptor modulators as multifunctional medicines. 2. Clinical considerations and new agents. J. Med. Chem. 46, 1081-1111 (2003).

3. Cipriani, A. et al. Comparative efficacy and acceptability of 21 antidepressant drugs for the acute treatment of adults with major depressive disorder: a systematic review and network meta-analysis. Lancet 391, 1357-1366 (2018).

4. Trost, B. M. \& Fleming, I. Comprehensive Organic Synthesis (Pergamon Press, 1991).

5. Itami, K. \& Yoshida, J.-i Multisubstituted olefins: platform synthesis and applications to materials science and pharmaceutical chemistry. Bull. Chem. Soc. Jpn. 79, 811-824 (2006).

6. Flynn, A. B. \& Ogilvie, W. W. Stereocontrolled synthesis of tetrasubstituted olefins. Chem. Rev. 107, 4698-4745 (2007).

7. Wang, J. Stereoselective Alkene Synthesis (Springer, 2012). 
8. Fallis, A. G. \& Forgione, P. Metal mediated carbometallation of alkynes and alkenes containing adjacent heteroatoms. Tetrahedron 57, 5899-5913 (2001).

9. Alonso, F., Beletskaya, I. P. \& Yus, M. Transition-metal-catalyzed addition of heteroatom-hydrogen bonds to alkynes. Chem. Rev. 104, 3079-3160 (2004).

10. Moslin, R. M., Miller-Moslin, K. \& Jamison, T. F. Regioselectivity and enantioselectivity in nickel-catalysed reductive coupling reactions of alkynes. Chem. Commun. 4441-4449 (2007).

11. Marek, I., Chinkov, N. \& Banon-Tenne, D. Carbometallation Reactions (Wiley-VCH Verlag GmbH, 2008).

12. Chinchilla, R. \& Nájera, C. Chemicals from alkynes with palladium catalysts. Chem. Rev. 114, 1783-1826 (2014).

13. Greenhalgh Mark, D., Jones Alison, S. \& Thomas Stephen, P. Iron-catalysed hydrofunctionalisation of alkenes and alkynes. ChemCatChem 7, 190-222 (2014).

14. Sam, B., Breit, B. \& Krische Michael, J. Paraformaldehyde and mMethanol as $\mathrm{C} 1$ feedstocks in metal-catalyzed C-C cCouplings of p-unsaturated reactants: beyond hydroformylation. Angew. Chem. Int. Ed. 54, 3267-3274 (2014).

15. Jackson, E. P. et al. Mechanistic basis for regioselection and regiodivergence in nickel-catalyzed reductive couplings. Acc. Chem. Res. 48, 1736-1745 (2015).

16. Yoshida, H. Borylation of alkynes under base/coinage metal catalysis: some recent developments. ACS Catal. 6, 1799-1811 (2016).

17. Ghosh, A., Johnson, K. F., Vickerman, K. L., Walker, J. A. \& Stanley, L. M. Recent advances in transition metal-catalysed hydroacylation of alkenes and alkynes. Org. Chem. Front 3, 639-644 (2016).

18. Boyarskiy, V. P., Ryabukhin, D. S., Bokach, N. A. \& Vasilyev, A. V. Alkenylation of arenes and heteroarenes with alkynes. Chem. Rev. 116, 5894-5986 (2016).

19. Suess, A. M. \& Lalic, G. Copper-catalyzed hydrofunctionalization of alkynes. Synlett 27, 1165-1174 (2016)

20. Jordan, A. J., Lalic, G. \& Sadighi, J. P. Coinage metal hydrides: synthesis, characterization, and reactivity. Chem. Rev. 116, 8318-8372 (2016).

21. Zheng, Y. \& Zi, W. Transition-metal catalyzed enantioselective hydrofunctionalization of alkynes. Tetrahedron Lett. 59, 2205-2213 (2018).

22. Herath, A. \& Montgomery, J. Highly chemoselective and stereoselective synthesis of Z-eEnol sSilanes. J. Am. Chem. Soc. 130, 8132-8133 (2008).

23. Shi, S.-L. \& Buchwald, S. L. Copper-catalysed selective hydroamination reactions of alkynes. Nat. Chem. 7, 38 (2014).

24. Xue, F., Zhao, J., Hor, T. S. A. \& Hayashi, T. Nickel-catalyzed threecomponent domino reactions of aryl Grignard reagents, alkynes, and aryl halides producing tetrasubstituted alkenes. J. Am. Chem. Soc. 137, 3189-3192 (2015).

25. Wang, X., Nakajima, M., Serrano, E. \& Martin, R. Alkyl bromides as mild hydride sources in $\mathrm{Ni}$-catalyzed hydroamidation of alkynes with isocyanates. J. Am. Chem. Soc. 138, 15531-15534 (2016).

26. Liu, Z., Derosa, J. \& Engle, K. M. Palladium(II)-catalyzed regioselective synhydroarylation of disubstituted alkynes using a removable directing group. J. Am. Chem. Soc. 138, 13076-13081 (2016).

27. Kortman, G. D. \& Hull, K. L. Copper-catalyzed hydroarylation of internal alkynes: highly regio- and diastereoselective synthesis of 1,1-diaryl, trisubstituted olefins. ACS Catal. 7, 6220-6224 (2017).

28. Clarke, C., Incerti-Pradillos, C. A. \& Lam, H. W. Enantioselective nickelcatalyzed anti-carbometallative cyclizations of alkynyl electrophiles enabled by reversible alkenylnickel E/Z iIsomerization. J. Am. Chem. Soc. 138, 8068-8071 (2016).

29. Wille, U. Radical cascades initiated by intermolecular radical addition to alkynes and related triple bond systems. Chem. Rev. 113, 813-853 (2013)

30. Li, Z., García-Domínguez, A. \& Nevado, C. Pd-catalyzed stereoselective carboperfluoroalkylation of alkynes. J. Am. Chem. Soc. 137, 11610-11613 (2015).

31. Wang, F., Zhu, N., Chen, P., Ye, J. \& Liu, G. Copper-catalyzed trifluoromethylazidation of alkynes: efficient access to CF3-substituted azirines and aziridines. Angew. Chem. Int. Ed. 54, 9356-9360 (2015).

32. Xu, T. \& Hu, X. Copper-catalyzed 1,2-addition of $\alpha$-carbonyl iodides to alkynes. Angew. Chem. Int. Ed. 54, 1307-1311 (2015)

33. He, Y.-T., Li, L.-H., Wang, Q., Wu, W. \& Liang, Y.-M. Synthesis of $\beta$ difluoroalkylated acrylonitriles in the presence of copper powder. Org. Lett. 18, 5158-5161 (2016).

34. Li, Z., García-Domínguez, A. \& Nevado, C. Nickel-catalyzed stereoselective dicarbofunctionalization of alkynes. Angew. Chem. Int. Ed. 55, 6938-6941 (2016).

35. Domański, S. \& Chaładaj, W. A broadly applicable method for Pd-catalyzed carboperfluoro-alkylation of terminal and internal alkynes: a convenient route to tri- and tetrasubstituted olefins. ACS Catal. 6, 3452-3456 (2016).

36. Garcia-Dominguez, A., Muller, S. \& Nevado, C. Nickel-catalyzed intermolecular carbosulfonylation of alkynes via sulfonyl radicals. Angew. Chem. Int. Ed. 56, 9949-9952 (2017).

37. García-Domínguez, A., Li, Z. \& Nevado, C. Nickel-catalyzed reductive dicarbofunctionalization of alkenes. J. Am. Chem. Soc. 139, 6835-6838 (2017).
38. Stephenson, C. R. J., Yoon, T. P. \& MacMillan, D. W. C. Visible Light Photocatalysis in Organic Chemistry (Wiley-VCH, 2018).

39. Prier, C. K., Rankic, D. A. \& MacMillan, D. W. C. Visible light photoredox catalysis with transition metal complexes: applications in organic synthesis. Chem. Rev. 113, 5322-5363 (2013).

40. Chen, J.-R., Hu, X.-Q., Lu, L.-Q. \& Xiao, W.-J. Visible light photoredoxcontrolled reactions of N-radicals and radical ions. Chem. Soc. Rev. 45, 2044-2056 (2016).

41. Douglas, J. J., Sevrin, M. J. \& Stephenson, C. R. J. Visible light photocatalysis: applications and new disconnections in the synthesis of pharmaceutical agents. Org. Process Res. Dev. 20, 1134-1147 (2016).

42. Romero, N. A. \& Nicewicz, D. A. Organic photoredox catalysis. Chem. Rev. 116, 10075-10166 (2016)

43. Marzo, L., Pagire, S. K., Reiser, O. \& Konig, B. Visible-light photocatalysis: does it make a difference in organic synthesis? Angew. Chem. Int. Ed. 57, 10034-10072 (2018).

44. Tellis, J. C. et al. Single-electron transmetalation via photoredox/nickel dual catalysis: unlocking a new paradigm for $\mathrm{sp}^{3}-\mathrm{sp}^{2}$ cross-coupling. Acc. Chem. Res 49, 1429-1439 (2016).

45. Hopkinson, M. N., Tlahuext-Aca, A. \& Glorius, F. Merging visible light photoredox and gold catalysis. Acc. Chem. Res. 49, 2261-2272 (2016).

46. Gui, Y.-Y., Sun, L., Lu, Z.-P. \& Yu, D.-G. Photoredox sheds new light on nickel catalysis: from carbon-carbon to carbon-heteroatom bond formation. Org. Chem. Front 3, 522-526 (2016).

47. Fabry, D. C. \& Rueping, M. Merging visible light photoredox catalysis with metal catalyzed $\mathrm{C}-\mathrm{H}$ aActivations: on the role of oxygen and superoxide ions as oxidants. Acc. Chem. Res. 49, 1969-1979 (2016).

48. Cavalcanti, L. N. \& Molander, G. A. Photoredox catalysis in nickel-catalyzed cross-coupling. Top. Curr. Chem. 374, 39 (2016).

49. Twilton, J. et al. The merger of transition metal and photocatalysis. Nat. Rev Chem. 1, 0052 (2017).

50. Singh, K., Staig, S. J. \& Weaver, J. D. Facile sSynthesis of Z-alkenes via uphill catalysis. J. Am. Chem. Soc. 136, 5275-5278 (2014).

51. Metternich, J. B. \& Gilmour, R. A bio-inspired, catalytic $\mathrm{E} \rightarrow \mathrm{Z}$ isomerization of activated olefins. J. Am. Chem. Soc. 137, 11254-11257 (2015).

52. Metternich, J. B. \& Gilmour, R. One photocatalyst, n activation modes strategy for cascade catalysis: emulating coumarin biosynthesis with (-)-rRiboflavin. J. Am. Chem. Soc. 138, 1040-1045 (2016).

53. Molloy, J. J., Metternich, J. B., Daniliuc, C. G., Watson, A. J. B. \& Gilmour, R. Contra-thermodynamic, photocatalyticThermodynamic, Photocatalytic E-->Z isomerization of styrenyl boron species: vectors to facilitate exploration of twodimensional chemical space. Angew. Chem. Int. Ed. 57, 3168-3172 (2018).

54. Faßbender, S. I., Metternich, J. B. \& Gilmour, R. Spatiotemporal control of pre-existing alkene geometry: a bio-inspired route to 4-trifluoromethyl-2Hchromenes. Org. Lett. 20, 724-727 (2018).

55. Metternich Jan, B. et al. Covalent iImmobilization of (-)-riboflavin on polymer functionalized silica particles: application in the photocatalytic $\mathrm{E} \rightarrow \mathrm{Z}$ isomerization of polarized alkenes. Chem. Eur. J. 24, 4228-4233 (2018).

56. Pearson, C. M. \& Snaddon, T. N. Alkene photo-isomerization inspired by vision. ACS Cent. Sci. 3, 922-924 (2017).

57. Deng, H.-P., Fan, X.-Z., Chen, Z.-H., Xu, Q.-H. \& Wu, J. Photoinduced nickelcatalyzed chemo- and regioselective hydroalkylation of internal alkynes with ether and amide $\alpha$-hHetero C(sp3)-H bBonds. J. Am. Chem. Soc. 139, 13579-13584 (2017).

58. Hou, J. et al. Visible-light-driven alkyne hydro-/carbocarboxylation using $\mathrm{CO}_{2}$ via iridium/cobalt dual catalysis for divergent heterocycle synthesis. J. Am. Chem. Soc. 140, 5257-5263 (2018).

59. Till, N. A., Smith, R. T. \& MacMillan, D. W. C. Decarboxylative hydroalkylation of alkynes. J. Am. Chem. Soc. 140, 5701-5705 (2018).

60. Matsui, J. K., Lang, S. B., Heitz, D. R. \& Molander, G. A. Photoredox-mediated routes to radicals: the value of catalytic radical generation in synthetic methods development. ACS Catal. 7, 2563-2575 (2017).

61. Zhang, X. \& MacMillan, D. W. C. Alcohols as latent coupling fragments for metallaphotoredox catalysis: $\mathrm{sp}^{3}-\mathrm{sp}^{2}$ cross-coupling of oxalates with aryl halides. J. Am. Chem. Soc. 138, 13862-13865 (2016).

62. Tellis, J. C., Primer, D. N. \& Molander, G. A. Single-electron transmetalation in organoboron cross-coupling by photoredox/nickel dual catalysis. Science 345, 433-436 (2014).

63. Zuo, Z. et al. Merging photoredox with nickel catalysis: CCoupling of a-carboxyl $\mathrm{sp}^{3}$-carbons with aryl halides. Science 345, 437-440 (2014).

64. Nawrat, C. C., Jamison, C. R., Slutskyy, Y., MacMillan, D. W. C. \& Overman, L. E. Oxalates as activating groups for alcohols in visible light photoredox catalysis: formation of quaternary centers by redox-neutral fragment coupling. J. Am. Chem. Soc. 137, 11270-11273 (2015).

65. Lowry, M. S. et al. SSingle-layer electroluminescent devices and photoinduced hydrogen production from an ionic iridium(III) C complex. Chem. Mater. 17, 5712-5719 (2005) 
66. Cheung, C. W., Zhurkin, F. E. \& Hu, X. Z-selective olefin synthesis via ironcatalyzed reductive coupling of alkyl halides with terminal arylalkynes. J. Am. Chem. Soc. 137, 4932-4935 (2015).

67. Liu, J., Ren, Q., Zhang, X. \& Gong, H. Preparation of vinyl arenes by nickelcatalyzed reductive coupling of aryl halides with vinyl bromides. Angew. Chem. Int. Ed. 55, 15544-15548 (2016).

68. Durandetti, M., Devaud, M. \& Perichon, J. Investigation of the reductive coupling of aryl halides and/or ethylchloroacetate electrocatalyzed by the precursor $\mathrm{NiX}_{2}$ (bpy) with $\mathrm{X}$ - $=\mathrm{Cl}$-, $\mathrm{Br}$ - or $\mathrm{MeSO}_{3}$ - and bpy = 2,2'-dipyridyl. New J. Chem. 20, 659-667 (1996).

69. Heitz, D. R., Tellis, J. C. \& Molander, G. A. Photochemical nickel-catalyzed $\mathrm{C}-\mathrm{H}$ aArylation: synthetic scope and mechanistic investigations. J. Am. Chem. Soc. 138, 12715-12718 (2016).

70. Primer, D. N. \& Molander, G. A. Enabling the cross-coupling of tertiary organoboron nucleophiles through radical-mediated alkyl transfer. J. Am. Chem. Soc. 139, 9847-9850 (2017).

71. Sun, S.-Z. \& Martin, R. Nickel-catalyzed umpolung arylation of ambiphilic abromoalkyl boronic esters. Angew. Chem. Int. Ed. 57, 3622-3625 (2018).

\section{Acknowledgements}

We thank the National Natural Science Foundation of China (21702029), and the "Thousand Plan" Youth Program and the Shanghai Sailing Program (17YF1400100) for financial support. We thank Prof. David MacMillan (Princeton University) for discussions, and Prof. Chao Zheng (Shanghai Institute of Organic Chemistry, CAS) for DFT calculations.

\section{Author contributions}

L.C. conceived and designed the project. L.C. and L.G. designed the experiments. L.G., S. F., S.Z. and H.L. performed the experiments and analyzed the data. L.C. prepared the manuscript.

\section{Additional information}

Supplementary Information accompanies this paper at https://doi.org/10.1038/s41467 018-06904-9.

Competing interests: The authors declare no competing interests.

Reprints and permission information is available online at http://npg.nature.com/ reprintsandpermissions/

Publisher's note: Springer Nature remains neutral with regard to jurisdictional claims in published maps and institutional affiliations.

\section{(c) (i)}

Open Access This article is licensed under a Creative Commons Attribution 4.0 International License, which permits use, sharing, adaptation, distribution and reproduction in any medium or format, as long as you give appropriate credit to the original author(s) and the source, provide a link to the Creative Commons license, and indicate if changes were made. The images or other third party material in this article are included in the article's Creative Commons license, unless indicated otherwise in a credit line to the material. If material is not included in the article's Creative Commons license and your intended use is not permitted by statutory regulation or exceeds the permitted use, you will need to obtain permission directly from the copyright holder. To view a copy of this license, visit http://creativecommons.org/ licenses/by/4.0/.

(C) The Author(s) 2018 